Musées, Patrimoine et Culture scientifiques et techniques

$146 \mid 2013$

mars-avril 2013

\title{
La complétude des spécimens et les processus de conservation
}

Alexis Martin et Jacques Cuisin

\section{OpenEdition \\ Journals}

Édition électronique

URL : http://journals.openedition.org/ocim/1210

DOI : $10.4000 /$ ocim. 1210

ISSN : 2108-646X

Éditeur

OCIM

Édition imprimée

Date de publication : 1 mars 2013

Pagination : $19-28$

ISSN : 0994-1908

Référence électronique

Alexis Martin et Jacques Cuisin, "La complétude des spécimens et les processus de conservation», La Lettre de l'OCIM [En ligne], 146 | 2013, mis en ligne le 01 mars 2015, consulté le 02 mai 2019. URL http://journals.openedition.org/ocim/1210; DOI : 10.4000/ocim.1210 


\section{La complétude des spécimens et les processus de conservation}

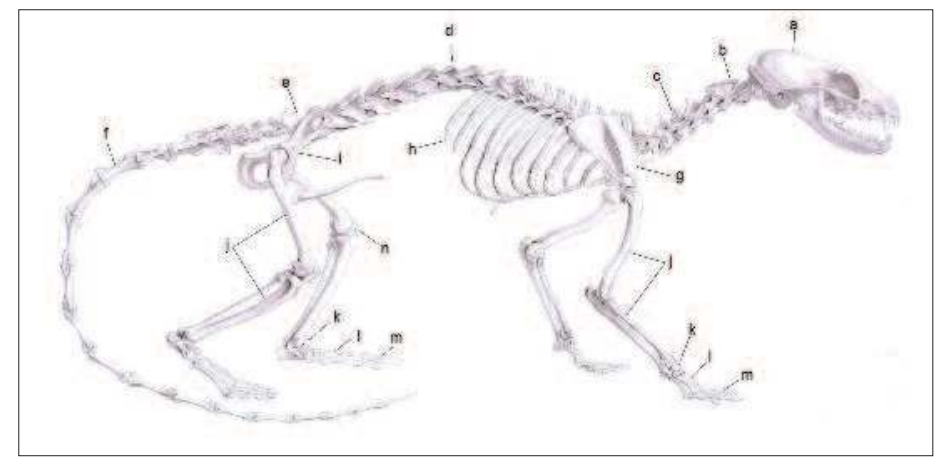

Organisation générale du squelette et localisation des parties anatomiques (source de l'image : infographie d'après "Mammifères Pl. 4 », Atlas Zoologique du Voyage de la corvette La Bonite, 1853,

Wikimedia Commons)

* Alexis Martin est chargé de projet au sein de l'UMR BOREA du Muséum national d'Histoire naturelle alexis.martin@mnhn.fr Jacques Cuisin est responsable de la plateforme de Préparation/Restauration au Muséum national d'Histoire naturelle cuisin@mnhn.fr

\section{Alexis Martin et Jacques Cuisin *}

À partir de l'analyse statistique des données $d^{\prime}$ inventaire des collections anatomiques $d u$ Muséum national d'Histoire naturelle, cette contribution propose une étude des facteurs de conservation qui affectent la complétude des spécimens dans les collections ostéologiques.

Dans le domaine de l'Histoire naturelle, la notion de « complétude » répond à l'enjeu d'exploitabilité même des collections de spécimens. L'utilisation scientifique des animaux naturalisés ou préparés de diverses manières s'inscrit dans l'héritage scientifique de l'anatomie comparée de la fin du XVIII e siècle, qui fait de l'étude des « séries » d'objets, plus que des objets eux-mêmes, le socle de l'interprétation rationaliste de la diversité du vivant.

La nécessité de pouvoir s'appuyer sur des séries « complètes » est une contrainte incontournable, qu'il s'agisse de travaux de systématique, de biogéographie, d'archéozoologie, de paléontologie ou d'écologie. Nous pouvons citer par exemple la nécessité pour l'écologue de posséder en collection des représentants de tous les taxons présents dans une même aire d'étude, ou, à l'inverse, de posséder pour un même taxon des spécimens en provenance de toute son aire de répartition. L'importance de la complétude se révèle également pour le systématicien dont le travail s'appuiera nécessairement sur l'étude de séries exhaustives de spécimens de tous les taxons connus au sein d'un même groupe classifié.

La complétude peut également s'évaluer à l'échelle du spécimen du point de vue anatomique. Ce facteur est déterminant pour les collections ostéologiques. 
Composé de plusieurs centaines d'os, les squelettes de vertébrés impliquent pour le conservateur des enjeux tout à fait spécifiques. Si les objets individuels, les os, sont relativement robustes et physiquement faciles à conserver, il n'en est pas de même pour la dimension « documentaire » qu'induit leur mise en cohérence. Séparé du reste du squelette, un os perdu n'aura ainsi plus aucun intérêt car il sera impossible d'en tirer la moindre information scientifique exploitable. Un squelette trop partiel verra également son exploitabilité amoindrie. Ces enjeux sont d'autant plus importants que les risques de pertes sont multiples dans la vie d'une collection (Bello et al., 2003).

Le travail que nous présentons s'appuie sur l'étude des collections ostéologiques du Muséum National d'Histoire Naturelle à Paris. Nous avons analysé statistiquement une partie des résultats d'inventaires anatomiques exhaustifs que nous avons réalisés dans deux collections historiques de l'établissement. À partir de ces résultats, nous proposons une interprétation et nous essayons de comprendre quels sont les facteurs de conservation qui peuvent permettre d'expliquer l'intégrité anatomique des spécimens.

\section{Hypothèses de travail}

La première hypothèse que nous souhaitons tester est celle d'une corrélation entre l'ancienneté des squelettes et leur complétude. Il est raisonnable de supposer que plus un squelette sera ancien, moins il sera complet du fait de l'accumulation dans le temps des risques de pertes d'éléments, lors des consultations ou lors des travaux de conservation eux-mêmes (déplacements, reconditionnements).

Nous souhaitons également vérifier un lien éventuel entre la dimension physique des squelettes et le degré de complétude. Deux présupposés peuvent être opposés l'un à l'autre. Un squelette de plus grande taille pourrait avoir une plus grande probabilité d'être plus complet, ses constituants étant moins faciles à égarer. À l'inverse, il est raisonnable d'envisager qu'un squelette de plus petite taille sera en règle générale globalement plus complet qu'un squelette de grande taille, car plus facilement et plus souvent rassemblé en un conditionnement unique garantissant son unité de rangement.

La troisième hypothèse est celle du lien entre le mode de préparation et le degré de complétude. Deux typologies existent. La préparation dite en « connexion ligamentaire » est obtenue par un long et fin travail de dissection au scalpel. Le préparateur retire toutes

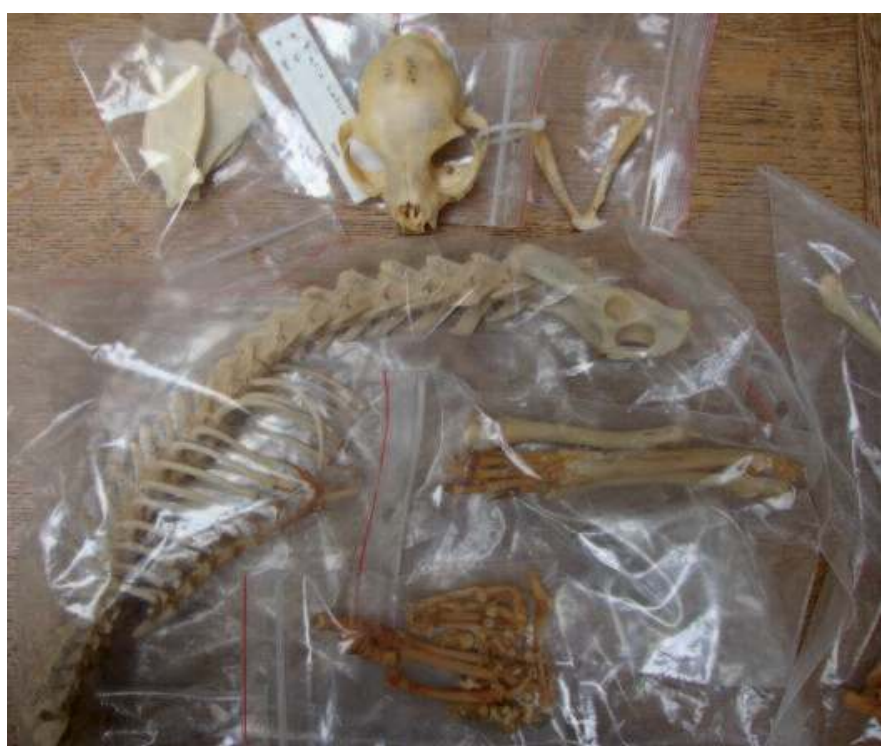

Un squelette incomplètement préparé, dont certains segments ont été laissés en connexion ligamentaires. Le risque de perte de petits éléments est moindre, celui de bris est augmenté par la rigidité de l'ensemble, la place de stockage nécessaire augmente, ici à cause de la colonne vertébrale entièrement connectée. (c) J. Cuisin

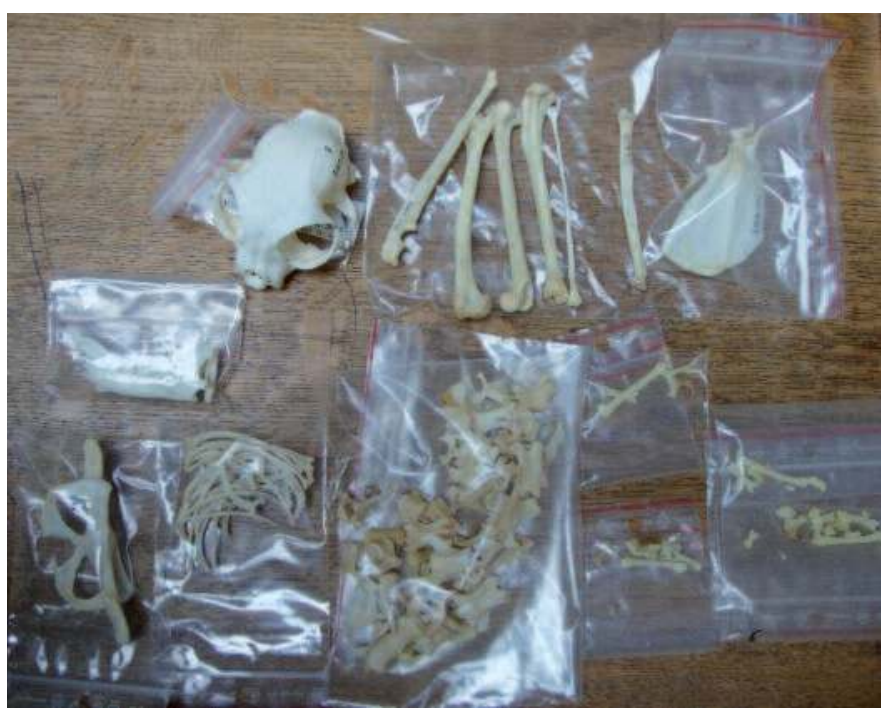

Un squelette en déconnexion ligamentaire, aussi dit « en vrac ». Les différents éléments, séparés, se prêtent plus facilement à la perte. Le bris est plus rare, la place de stockage est réduite.

๑ J. Cuisin

les parties carnées d'un cadavre frais, et conserve le squelette avec tous ses tissus connectifs. Les os restent alors tous reliés entre eux par les ligaments, séchés une fois la préparation terminée. Â l'inverse, il est possible de préparer un squelette « en vrac ». Le processus de préparation fait intervenir la digestion 
enzymatique des tissus et permet de ne conserver que les os. Tous les tissus mous, y compris les ligaments, sont détruits. Les os sont alors entièrement désolidarisés les uns des autres. L'observation des spécimens permet de voir que les squelettes en connexion ligamentaire sont en général, et logiquement, globalement plus complets que leurs homologues en vrac. Nous souhaitons vérifier l'hypothèse et mesurer le phénomène d'un point de vue statistique.

L'étude a également pour objectif de vérifier la persistance et l'incidence des différents facteurs susceptibles d'affecter la complétude (anatomie, historique conservatoire, dimension des spécimens), au sein de collections de taxons différents, toutefois suffisamment proches pour être comparables. Enfin, l'étude a pour but d'analyser tous ces facteurs à l'échelle de la complétude globale des squelettes ainsi qu'à celle de différentes sous-régions anatomiques cohérentes. Nous souhaitons alors vérifier si des parties sont plus ou moins sensibles que d'autres aux différents facteurs testés.

\section{Matériels et méthodes}

Létude est fondée sur l'analyse statistique des résultats d'inventaire de deux sections complètes des collections ostéologiques des laboratoires d'Anatomie Comparée et de Zoologie Mammifères et Oiseaux du Muséum national d'Histoire naturelle (Martin, 2005). L'inventaire a été conduit dans le cadre du chantier de rénovation des collections de l'établissement, impliquant une opération conjointe de reconditionnement individuel des spécimens (Cuisin et al., 2006).

\section{Les données}

Parmi les données disponibles que nous avons recueillies lors du chantier, nous avons retenu pour notre analyse celles des squelettes complets ou partiels de la collection des mammifères du groupe des carnivores dits "de petite et de moyenne taille ", ainsi que celles de la collection des lacertiliens (lézards). Les nombreux spécimens uniquement constitués du crâne ne sont pas retenus dans le corpus d'étude.

Les deux séries analysées présentent l'intérêt d'être chacune homogène en tant qu'entité de collection, du point de vue de leur passé conservatoire et de leur utilisation. De plus, les squelettes de ces deux groupes sont distribués suivant des classes de tailles similaires. Ils sont également comparables au plan de la complexité et de la composition anatomique. Enfin, les utilisateurs de ces collections sont différents étant donné le cloisonnement disciplinaire lié à la systématique.
La comparaison des analyses menées indépendamment sur ces deux suites de données permettra ainsi de corroborer les différentes interprétations ou au contraire de les différencier.

Nous n'avons pas retenu d'autres jeux de données disponibles, constitués lors d'inventaires complets que nous avons effectués en parallèle pour les besoins du chantier de rénovation. Les collections de mammifères du groupe des artiodactyles sont composées de spécimens de très grande taille et impliquent des conditions de conditionnement et de stockage totalement différentes. Les collections d'ophidiens (serpents) sont constituées de squelettes de dimensions comparables aux lacertiliens, et partagent un même historique de conservation et d'exploitation. Néanmoins, la complexification ostéologique différente d'un groupe à l'autre ne permet pas de les comparer suivant leur complétude : les lacertiliens sont des animaux tétrapodes, tandis que les squelettes des ophidiens sont constitués d'un crâne et d'un squelette axial.

\section{L'examen des collections}

Lors de l'inventaire, chaque squelette fait l'objet d'un examen complet. Nous enregistrons et vérifions le taxon, ainsi que le détail ostéologique en déterminant tous les os présents (Martin, 2005). Nous notons la présence ou l'absence de chacun pour l'intégralité des os crâniens externes, les deux premières vertèbres cervicales, le sacrum, les os des ceintures scapulaire et pelvienne, les rotules, ainsi que les os dits « longs » des quatre membres. Pour certaines régions du squelette nous effectuons des regroupements typologiques et spatialisés, et nous notons la présence en totalité, la présence partielle ou l'absence : les vertèbres cervicales, dorsales et caudales, les côtes, ainsi que les basipodes (carpes et tarses), métapodes (métacarpes et métatarses) et acropodes (phalanges) de chaque membre.

Les données non biologiques utiles à la conservation et à la gestion des collections sont également recueillies lors de l'inventaire. Nous retiendrons parmi ces informations trois variables dont la relation avec la complétude sera analysée.

La référence du produit utilisé pour le reconditionnement de chaque spécimen est ainsi enregistrée. En connaissant le volume de chacun des conditionnements normalisés que nous utilisons, il nous est alors possible d'obtenir une approximation du volume individuel des squelettes. La normalisation nous permet en outre de profiter d'une série de classes de tailles régulières et identiques dans les deux collections analysées, autorisant ainsi les comparaisons. 


\section{Herpestidae}

Atilax paludinosus

Bdeogale crassicauda

Bdeogale nigripes

Crossarchus obscurus

Galidia elegans

Galidictis vittata

Herpestes auropunctatus

Herpestes brachyurus

Herpestes edwardsii

Herpestes gracilis

Herpestes griseus

Herpestes ichneumon

Herpestes javanicus

Herpestes pharaonis

Herpestes sanguineus

Herpestes smithii

Herpestes urva

Mungos mungo

Paradoxurus javanicus

Salanoia concolor

Suricata suricatta

\section{Mustelidae}

Aonyx capensis

Aonyx cinera

Arctonyx collaris

Conepatus chinga

Eira barbara

Enhydra lutris

Galictis cuja

Galictis vittata

Gulo gulo

Ictonyx libyca

Ictonyx striatus

Lontra canadensis

Lontra felina

Lontra longicaudis

Lontra provocax

Lutra brasiliensis

Lutra capensis

Lutra lutra

Lutra maculicollis

Lutra sumatrana

Lyncodon patagonicus

Martes americana

Martes foina

Martes martes

Martes zibellina

Meles meles

Mellivora capensis

Melogale moschata

Melogale orientalis

Mephitis mephitis

Mustela erminea

Mustela frenata

Mustela itatsi

Mustela lutreola

Mustela nivalis
Mustela patagonica

Mustela putorius

Mustela rixosa

Mustela streatori

Mustela vison

Mydaus javanensis

Spilogale putorius

Taxidea taxus

Procyonidae

Bassariscus astutus

Bassariscus rusa

Cercoleptes caudivolvulus

Nasua narica

Nasua nasua

Potos flavus

Procyon cancrivorus

Procyon lotor

\section{Viverridae}

Arctictis binturong

Civettictis civetta

Cryptoprocta ferox

Cynogale bennettii

Genetta genetta

Genetta genettoïdes

Genetta maculata

Genetta pardina

Genetta servalina

Genetta tigrina

Hemigalus derbyanus

Hemigalus hardwickei

Nandinia binotata

Paguma larvata

Paradoxurus hermaphroditus

Paradoxurus larvatus

Paradoxurus quadrilineatus

Prionodon linsang

Viverra civetta

Viverra civettina

Viverra tangalunga

Viverra zibetha

Viverricula indica

\section{Lacertilia}

Calotes cristatellus

Calotes jubatus

Chalcides lineatus

Lygosoma tetradactylum

Ophisaurus apus

Ophisaurus ventralis

Peropus mutilatus

Phelsuma madagascariensis

Phrynosoma modestome

Taenilata ornata

Uroplates fimbriatus

\section{Agamidae}

Agama atra

Agama bibroni

Agama cristata

Agama stellio
Amphibolorus barbatus

Amphibolurus barbatus

Amphibolurus caudicinctus

Amphibolurus muricatus

Calotes mystaceus

Calotes versicolor

Draco fimbriatus

Draco lineatus

Draco volans

Hypsilurus spinipes

Leiolepis belliana

Physignathus cochinchinensis

Trapelus mutabilis

Uromastyx acanthinurus

Uromastyx hardwickii

\section{Anguidae}

Anguis fragilis

Gerrhonotus multicarinatus

Ophiodes striatus

Ophisaurus apodus

Pseudopus apodus

Chamaeleonidae

Chamaeleo bifidus

Chamaeleo chamaeleon

Chamaeleo cristatus

Chamaeleo dilepis

Chamaeleo ellioti

Chamaeleo gracilis

Chamaeleo johnstoni

Chamaeleo oweni

Chamaeleo pardalis

Chamaeleo parsonii

Chamaeleo senegalensis

Chamaeleon dilepis

Chamaeleon oustaleti

\section{Cordylidae}

Cordylus vittifer

Gerrhausaurus major

Zonorus giganteus

Zonurus cordylus

\section{Gekkonidae}

Gekko gecko

Hemidactylus frenatus

Hemidactylus mabouia

Hemidactylus turcicus

Oedura tryoni

Phelsuma cepedianum

Tarentola mauritanica

\section{Gerrhosauridae}

Zonosaurus madagascariensis

Helodermatidae

Heloderma suspectum

Iguanidae

Anolis carolinensis

Anolis equestris

Basilicus basilicus

Basilicus vittatus

Ctenosaura acanthura
Cyclura carinata

Cyclura cornuta

Dipsosaurus dorsalis

Iguana delicatissima

Iguana iguana

Phrynosoma cornutum

Phrynosoma coronatum

Polychrus marmoratus

Sceloporus occidentalis

Lacertidae

Algyroides fitzingeri

Eremias arguta

Eremias guttulata

Lacerta agilis

Lacerta lepida

Lacerta muralis

Lacerta viridis

Opluridae

Chalarodon madagascariensis

\section{Scincidae}

Ablepharus walbergi

Acontias meleagris

Acontias meleagui

Acontias plumbeus

Chalcides chalcides

Chalcides ocellatus

Egernia cunninghami

Eumeces algeriensis

Feylinia currori

Mabuya aurata

Mabuya maculilabris

Mabuya multifasciata

Mabuya varia

Mabuya vittata

Macroscincus cocteaui

Scincus scincus

Tiliqua nigrolutea

Tiliqua scincoides

Trachydosaurus rugosus

\section{Teiidae}

Ameiva ameiva

Ameiva chrysolaema

Neusticurus rudis

Tupinambis nigropunctatus

Tupinambis teguixin

\section{Varanidae}

Varanus ammodytes

Varanus bengalensis

Varanus exanthematicus

Varanus flavescens

Varanus gouldii

Varanus griseus

Varanus monitor

Varanus niloticus

Varanus ocellatus

Varanus rudicollis

Varanus salvator

Varanus varius 
Le numéro de catalogue de chaque spécimen est également enregistré. Cet identifiant millésimé est composé de l'année d'inscription du squelette en collection et d'un incrément régulier d'une unité pour chaque spécimen rentré la même année. Il est ainsi possible, grâce à cet identifiant, d'extraire l'année d'entrée de chaque squelette et de posséder une variable liée à son ancienneté en tant qu'objet de collection.

Enfin, le type de préparation est mentionné lors de l'inventaire : nous spécifions la présence ou non des connexions ligamentaires.

\section{Le traitement des données}

Au total, l'étude implique l'examen et la détermination d'environ 140000 pièces ostéologiques, provenant de squelettes inscrits au catalogue entre les années 1840 et 2006 .

Les données recueillies sur les carnivores constituent une matrice de 327 spécimens qualifiés par 72 descripteurs ostéologiques de présence/absence, un taxon et trois variables qualitatives, soit l'enregistrement de 24852 variables. Les données recueillies sur les lacertiliens constituent une matrice similaire de 397 spécimens qualifiés par 106 descripteurs anatomiques, soit l'enregistrement de 43670 variables.

Au sein de chaque matrice et pour chaque spécimen, nous calculons alors un pourcentage de complétude de quatre régions anatomiques : le crâne, les ceintures, les os longs et les autopodes (ensemble formé des tarses/ carpes, métatarses/métacarpes et des phalanges).

Pour ces derniers, par sous-groupe anatomique (carpes/tarses, métacarpes/métatarses, phalanges) et par membre (antérieur et postérieur, droit et gauche), nous attribuons $0 \%$ en cas «d'absence », $100 \%$ en cas de " présence complète » et $50 \%$ en cas de "présence partielle». Le pourcentage de complétude des autopodes est ensuite obtenu par une moyenne des 12 valeurs enregistrées (trois sous-groupes anatomiques pour quatre membres).

Ce pourcentage contient donc l'approximation de l'attribution de $50 \%$ aux autopodes partiels. Mais l'approximation n'amplifie ni ne minimise la complétude à l'échelle du lot de données, car on lui attribue une valeur centrale. Les valeurs de « $0 \%$ » et « $100 \%$ », qui concernent en outre la grande majorité des spécimens, sont des valeurs exactes.

Pour le crâne, les ceintures et les os longs, le pourcentage de complétude est un indice exact, l'inventaire étant conduit de façon exhaustive, avec un enregistrement os par os.

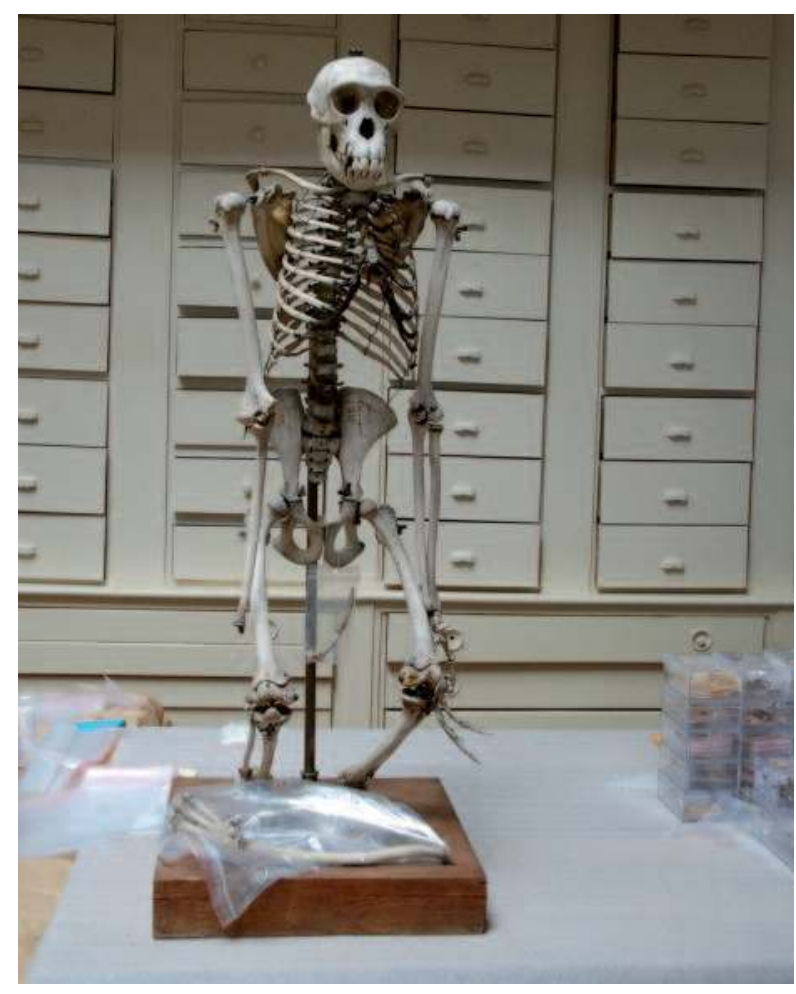

Squelette monté. Ce type de préparation se prête à une érosion des extrémités, qui affecte la complétude.

() J. Cuisin

\section{L'analyse statistique}

Le pourcentage de complétude globale de chaque squelette est obtenu ensuite par la moyenne des complétudes des quatre différentes régions, qui contribuent donc à égalité au calcul. L'approximation pour l'enregistrement des autopodes partiels introduit une erreur maximale théorique de plus ou moins $4 \%$ dans le calcul de la complétude globale (l'autopode est l'extrémité des membres chez les vertébrés tétrapodes, qui s'organise en 3 parties : basi-, méta- et acropode, soit tarse ou carpe, métacarpiens ou -tarsiens, et phalanges). Pour limiter le biais potentiel à ce chiffre, nous faisons le choix de ne pas conserver dans l'analyse l'enregistrement des côtes et des vertèbres, également approximé lors de l'inventaire pour des questions de faisabilité technique.

Nous observons ensuite la distribution de chaque série de données et effectuons des analyses statistiques à l'aide du logiciel R (R Development Core Team, 2008). L'étude préalable des effectifs, de la normalité et de la variance des distributions nous permet de choisir les tests statistiques appropriés.

Pour tester l'influence du facteur «ancienneté » sur la complétude, nous testons au sein de chaque série 
la corrélation linéaire entre l'année d'inscription au catalogue et le pourcentage de complétude globale de chaque spécimen.

Pour tester l'influence du facteur « volume », nous testons au sein de chaque série la corrélation entre la dimension du conditionnement et le pourcentage de complétude globale.

Suivant leur réussite, les tests de corrélation sont déclinés aux sous-régions anatomiques définies plus haut. Étant donné l'utilisation de variables discontinues comme facteurs, nous retenons l'utilisation du test de corrélation de Spearman (Spearman, 1910).

Pour tester l'influence du facteur "préparation », nous comparons entre elles au sein de chaque taxon les distributions de pourcentages de complétude selon la présence ou non des connexions ligamentaires. La taille des échantillons nous permet d'utiliser le test de Student (Gosset, 1908).

Nous testons l'hypothèse unilatérale d'une complétude supérieure chez les spécimens en connexion ligamentaire, au niveau global et dans chacune des sous-régions du squelette.

Le test est ajusté selon l'égalité ou non de la variance des distributions comparées.

\section{Résultats}

\section{Influence du facteur année}

Les données ne permettent pas d'observer une tendance générale dans l'évolution de la complétude anatomique actuelle des spécimens selon leur année d'inscription en collection, que ce soit pour les carnivores ou pour les lacertiliens.

Cette observation se trouve en outre corroborée par l'analyse statistique. Les résultats des tests indiquent en effet, avec une probabilité d'erreur extrêmement faible dans les deux cas, qu'il n'existe pas de corrélation significative liant ancienneté de l'inscription en collection et complétude des squelettes. La valeur de la p-value obtenue pour chacun des tests statistiques indique la valeur de cette probabilité, calculée sur la base de la structuration mathématique du jeu de données récolté. Pour les carnivores, la p-value de 0,7983 traduit le fait qu'il y a $79,83 \%$ de chances de se tromper, au plan statistique, en affirmant l'hypothèse que la complétude et l'année d'inscription sont corrélées mathématiquement. Pour les lacertiliens, les chances de se tromper en admettant l'hypothèse de cette même corrélation sont évaluées à 42,25\% (p-value $=0,4225)$. Classiquement, on considère dans le domaine scientifique qu'un test statistique est rejeté si la valeur de la p-value est supérieure à $5 \%$ soit 0,005 . Dans notre cas, l'hypothèse d'une corrélation peut donc être rejetée sans hésitation.

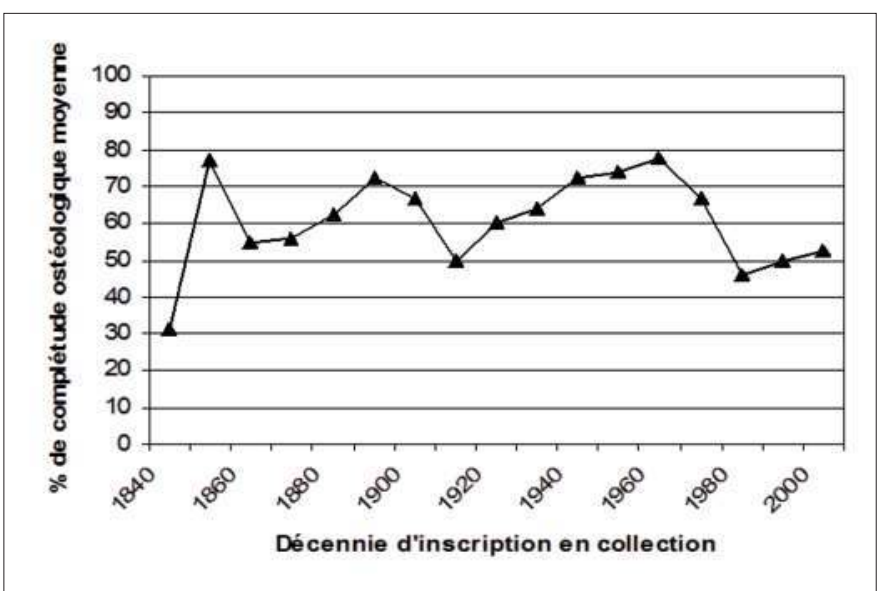

Complétude ostéologique des squelettes de carnivores suivant l'année de leur inscription en collection (les données sont représentées ici moyennées par décennie, pour plus de lisibilité).

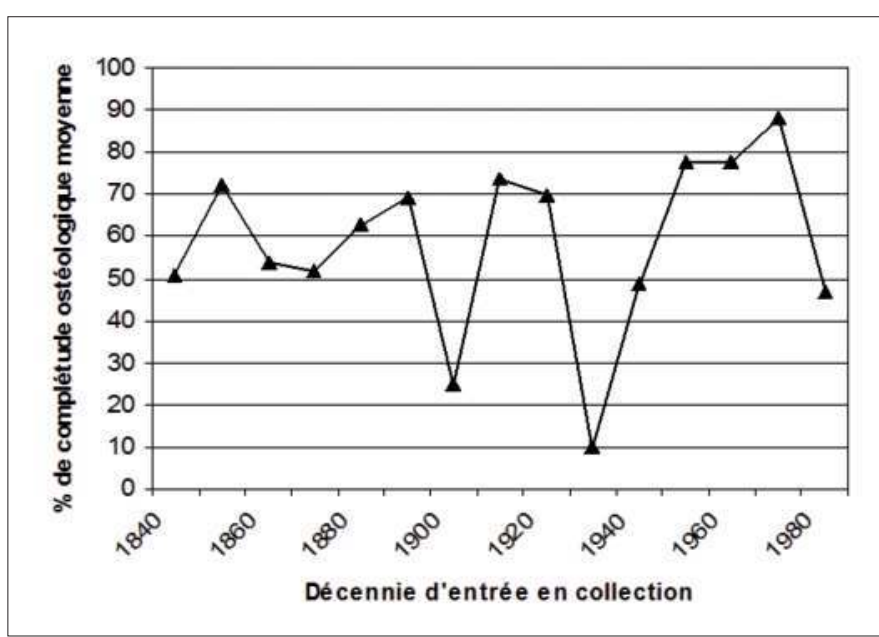

Complétude ostéologique des squelettes de lacertiliens suivant l'année de leur inscription en collection (les données sont représentées ici moyennées par décennie, pour plus de lisibilité).

\section{Influence du facteur volume}

Les données permettent en revanche d'observer une progression importante de la complétude anatomique globale avec l'accroissement du volume individuel des squelettes, pour les carnivores et pour les lacertiliens, quel que soit le type de préparation.

Chez les carnivores, la complétude varie ainsi en moyenne de 5 à $25 \%$ seulement avec les plus petits spécimens $\left(135 \mathrm{~cm}^{3}\right)$, selon la préparation, pour atteindre une fourchette de 63 à $80 \%$ avec les squelettes les plus grands $\left(8640 \mathrm{~cm}^{3}\right)$. Chez les lacertiliens, la complétude moyenne varie d'une fourchette basse de 25 à environ $50 \%$ chez les petits animaux, pour atteindre $75 \%$ avec les plus grands (en connexion ligamentaire). Les tests statistiques confirment en outre une corrélation linéaire importante et fortement significative, tant pour les carnivores que pour les lacertiliens. 
Déclinés à chacune des régions squelettiques, les tests montrent également que cette corrélation est similaire en force et en significativité pour les différentes parties de chaque spécimen, dans les deux ensembles taxinomiques.

Les valeurs de p-value des tests statistiques, pour tous les taxons et pour toutes les parties anatomiques, sont ainsi toujours proches de 0 . Elles indiquent que la probabilité de se tromper en affirmant l'hypothèse d'une corrélation entre volume et complétude est quasinulle dans les différents cas. Une valeur de p-value non seulement inférieure à $5 \%$ mais également proche de zéro témoigne en outre de la forte significativité du phénomène de corrélation révélé par le test.

La valeur de la statistique rho indique elle la force et la direction de la corrélation linéaire mise en évidence. Cet indice peut varier de zéro à un et être positif ou négatif. Pour les carnivores, les valeurs positives de rho de 0,4466 pour le test global et de 0,29 à 0,43 pour les sous-régions témoignent d'une corrélation positive et relativement forte : elles indiquent que la complétude augmente, et de manière relativement importante, quand ont augmente le volume du spécimen, pour chaque squelette dans son ensemble et pour chaque partie anatomique prise séparément. Les valeurs de rho permettent de conclure de manière identique pour les lacertiliens, avec un résultat du test global de 0,2936 et des valeurs de 0,22 à 0,37 pour le même test appliqué aux sous-groupes anatomiques.

\section{Influence du type de préparation}

L'observation montre que les spécimens préparés en connexion ligamentaire sont en moyenne globalement plus complets que les squelettes préparés en déconnexion, aussi bien chez les carnivores que chez les lacertiliens, quelle que soit la classe de taille concernée. Chez les carnivores et selon les classes de taille, la complétude des spécimens préparés en connexion ligamentaire est ainsi supérieure de 10 à $23 \%$ en moyenne. Chez les lacertiliens, la complétude des squelettes préparés en connexion peut être de quelques points supérieure à celle des spécimens en déconnexion, ou jusqu'à $27 \%$ supérieure en moyenne chez les animaux les plus petits.

L'analyse statistique appliquée à chaque région du squelette permet en outre d'affiner le résultat.

Les tests montrent ainsi que les squelettes préparés en connexion ligamentaire sont significativement plus complets au niveau des autopodes, des os longs et des ceintures, dans les deux groupes. Pour les carnivores et les lacertiliens, et pour ces différents sous-groupes anatomiques, la p-value proche de zéro indique que la probabilité de se tromper est quasi-nulle en affirmant l'hypothèse que la moyenne de la complétude des spécimens préparés en connexion ligamentaire est significativement supérieure.

Les crânes des lacertiliens sont également significativement plus complets lorsque les ligaments sont conservés. La p-value du test est proche de zéro et indique ainsi que la probabilité de se tromper est très faible si l'on retient l'hypothèse d'une complétude supérieure des crânes en préparation ligamentaire.

En revanche, la présence des connexions n'influence pas la complétude des crânes de carnivores. Le test entraîne une p-value de 0,1143 soit un risque de $11,43 \%$ de chances de se tromper en affirmant que les crânes des mammifères préparés en connexion ligamentaire sont significativement plus complets. Cette valeur est supérieure au seuil généralement admis de $5 \%$ et nous amène donc à rejeter l'hypothèse.

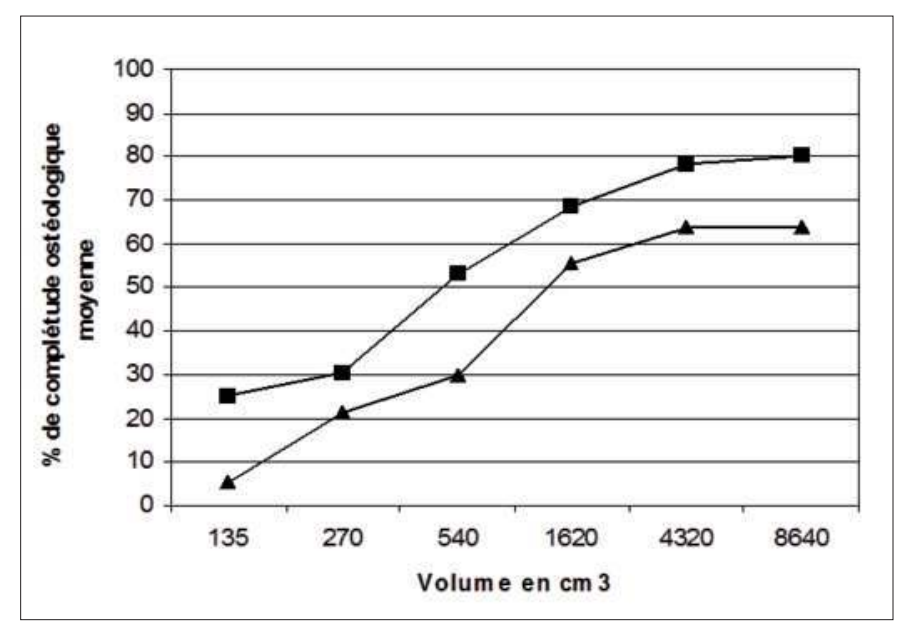

Complétude ostéologique des squelettes de carnivores suivant leur volume ( $\boldsymbol{\Delta}$ préparation en déconnexion,

préparation en connexion ligamentaire).

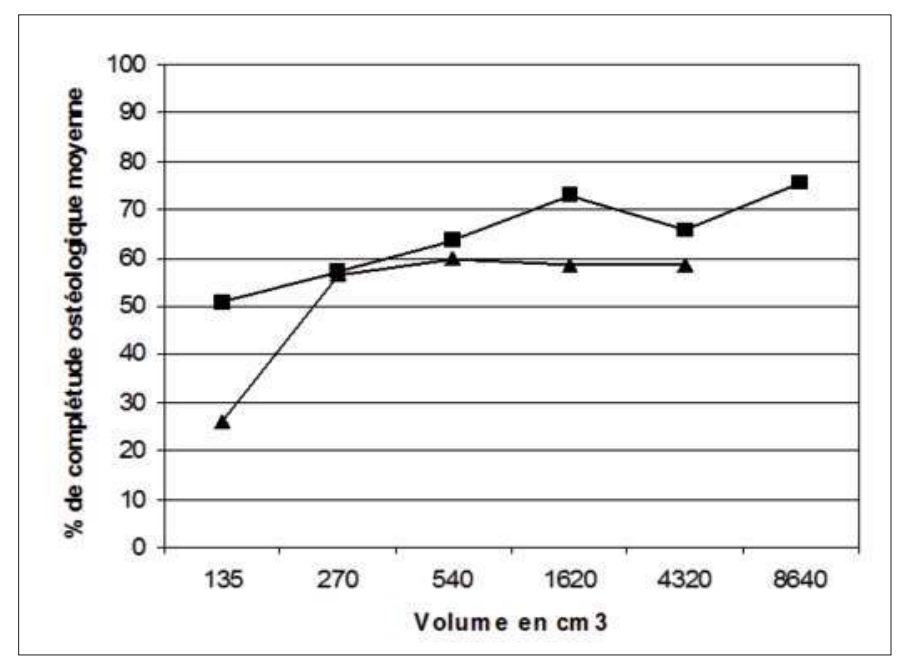

Complétude ostéologique des squelettes de lacertiliens suivant leur volume ( $\boldsymbol{\Delta}$ préparation en déconnexion,

préparation en connexion ligamentaire). 


\section{Discussion}

La complétude des squelettes ne semble pas liée à l'ancienneté de leur inscription en collection. Il est possible de formuler deux hypothèses à partir de ce constat.

Tout d'abord, les résultats pourraient indiquer que les pertes éventuelles et les dégradations liées à l'utilisation des spécimens lors des consultations ou aux travaux de conservation ne s'accumuleraient pas dans le temps de façon significative. Ceci peut témoigner soit de l'efficacité des mesures de conservation sur la collection étudiée, soit du fait que le facteur « ancienneté » serait en soi négligeable du point de vue de la complétude anatomique. Dans ce cas de figure, l'étude indiquerait que la mise à disposition et la consultation pourraient être considérées comme des étapes peu propices à la perte de constituants squelettiques.

La seconde hypothèse tient à celle d'un biais possible de l'étude. En effet, le facteur « ancienneté » est directement lié à la nature du spécimen : sauvage ou issu de captivité. L'implication en termes d'exploitation diffère : les spécimens anciens sont en général peu documentés, mais correspondent parfaitement à la demande des exploitants en systématique ou archéozoologie, par exemple. À l'inverse, les spécimens plus récents sont très bien documentés, mais peuvent être délaissés au motif qu'ils ont subi une période plus ou moins longue de captivité. Le facteur «quantité d'utilisations » est corrélé, quoique de manière non linéaire, à ce point. Il en résulterait que l'année d'inscription des spécimens au catalogue ne saurait pas forcément constituer une variable de contrôle pertinente pour mesurer l'impact dans le temps de l'exploitation de la collection, sur les pertes et les dégradations subies par les objets.

Le facteur «volume » est en revanche clairement identifié comme particulièrement déterminant : les squelettes de plus grande taille sont en moyenne beaucoup plus complets que les squelettes de petite taille, de façon très significative, et suivant une courbe de croissance régulière.

En outre, le facteur volume est déterminant indépendamment de toutes les autres mesures de contrôle retenues pour l'étude :

- les squelettes de plus grande taille sont plus complets quels que soient le taxon et le type de préparation,

- prises une à une, toutes les régions du squelette sont également significativement plus complètes chez les spécimens de plus grande taille.

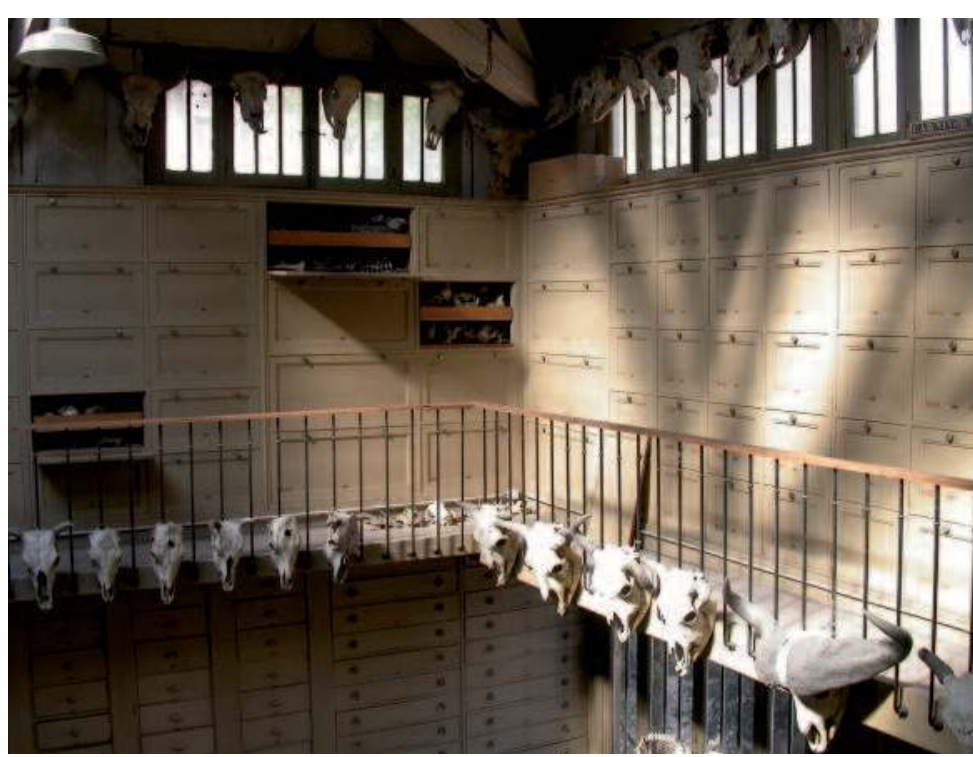

Vue générale de la salle dite " de la Girafe », où sont stockés la grande majorité des spécimens de petite et moyenne taille : tout est fait pour que la complétude des spécimens soit respectée.

๑ J. Cuisin

Le type de préparation impacte également la complétude. La conservation des connexions ligamentaires est ainsi déterminante et accroît sensiblement la complétude de toutes les régions squelettiques incluant une grande quantité d'éléments individuels. C'est le cas des crânes de lacertiliens et de toutes les régions post-crâniennes de tous les taxons. En revanche, le type de préparation n'est pas déterminant pour les crânes de carnivores, dont les os sont principalement soudés entre eux et constituent ainsi des ensembles unitaires. De plus, nous pouvons noter que la présence des connexions n'est pas significativement plus impactante pour la complétude des autopodes qu'elle ne l'est, par exemple, pour les os longs, pourtant moins nombreux et plus facilement identifiables.

Limportance de la présence des connexions ligamentaires semble donc moins liée à la complexité des parties du squelette qu'au simple fait de la connectivité ostéologique elle-même.

Enfin, la conjonction des résultats des tests statistiques nous permet de situer l'impact des facteurs significatifs au sein des étapes successives de la vie de la collection.

Si le test de corrélation entre le facteur année et la complétude s'était avéré être le seul significatif, les facteurs impactants n'auraient pu être identifiés, mais auraient pu être néanmoins situés après l'inscription des squelettes au catalogue. 
Si les risques de pertes liés à la taille du squelette ou l'absence des tissus connectifs étaient bien les facteurs explicatifs de la complétude, mais liés à la consultation des pièces, les phénomènes se seraient amplifiés dans le temps et auraient transmis un signal statistique mesurable par une corrélation significative entre le facteur année et la complétude.

Dans notre cas d'étude, sous réserve de la possibilité du biais évoqué au début de cette section de discussion, les facteurs volume et type de préparation ne s'accumuleraient pas dans le temps et impacteraient donc la complétude avant la consultation des squelettes, et donc avant leur inscription au catalogue.

Cet ensemble de résultats et d'observations permet alors d'envisager l'hypothèse globale suivante : l'étape de la préparation des spécimens est probablement la plus importante et la plus impactante pour la complétude anatomique des spécimens ; suivant cette hypothèse, la phase de conservation serait, pour le critère de complétude strictement, beaucoup moins sensible. Dans notre cas d'étude, il est ainsi très probable que le taux de complétude des spécimens s'explique plus facilement à l'issue du processus de préparation, plutôt qu'au moment de leur inscription en collection (les deux étapes peuvent être séparées d'un temps variable).

La préparation en connexion ligamentaire permet en effet de conserver une unité physique des squelettes, de la dissection jusqu'au conditionnement, et ainsi de limiter très fortement les risques de perte d'éléments. En outre, ce mode de préparation implique une attention importante et exclusive du préparateur pour son spécimen. Enfin, la conservation des connexions est obtenue par un travail de dissection qui limite « l'abandon » des spécimens préparés dans des solutions diverses de traitement, ainsi que la multiplication des étapes où le spécimen est déplacé.

En revanche, la préparation sans la conservation des connexions est obtenue par un processus rationalisé et standardisé, beaucoup plus complexe, impliquant la multiplication des étapes du traitement et du nombre de spécimens traités en une même session de travail.

Les phases de découpe, de traitement enzymatique, de rinçages multiples mettant en œuvre des filtres, de dégraissage, puis de séchage entraînent ainsi la multiplication des transferts de spécimens en cours de préparation d'un récipient à un autre, augmentant d'autant les risques de pertes d'os qu'ils sont désolidarisés après l'étape de traitement enzymatique.
Enfin la détectabilité des éléments selon leur taille sera le facteur le plus déterminant. Les risques de pertes d'objets lors de la préparation, en connexion ligamentaire ou non, seront d'autant plus grands que les squelettes seront de petite taille. L'étude et la vérification statistique démontrent, de façon solide, l'importance de ce critère.

La conséquence de cette donnée peut être traduite en approche préventive, non seulement de la conservation, mais aussi de la préparation : préparer en sorte que le produit fini sera le plus complet possible pour une valorisation optimale permet d'anticiper certaines étapes potentiellement plus risquées en termes de pertes. Il y a en effet une augmentation des risques de pertes d'une part lors des différents rinçages, qui font appel à de l'eau courante ou coulante, et d'autre part lors des périodes de séchage, lorsque le spécimen est laissé pendant un temps plus ou moins long de manière isolée mais à l'air libre et sans élément recouvrant.

À la suite de la préparation, le stockage est lui aussi source de pertes, surtout pour les spécimens de petite taille (vertébrés de moins de $12 \mathrm{~cm}$ de long lorsque vivants, par exemple). Les plus petits os ne sont pas aisément distinguables à l'œil nu (on pense à des carpes ou tarses de musaraignes, peu discernables de grains de graviers en première approche...). Il importe de prévenir cette perte par confusion via l'emploi de tubes ou de sachets transparents non électrostatiques et d'épaisseur suffisante, ces premiers contenants euxmêmes disposés dans des boîtes elles aussi en matière

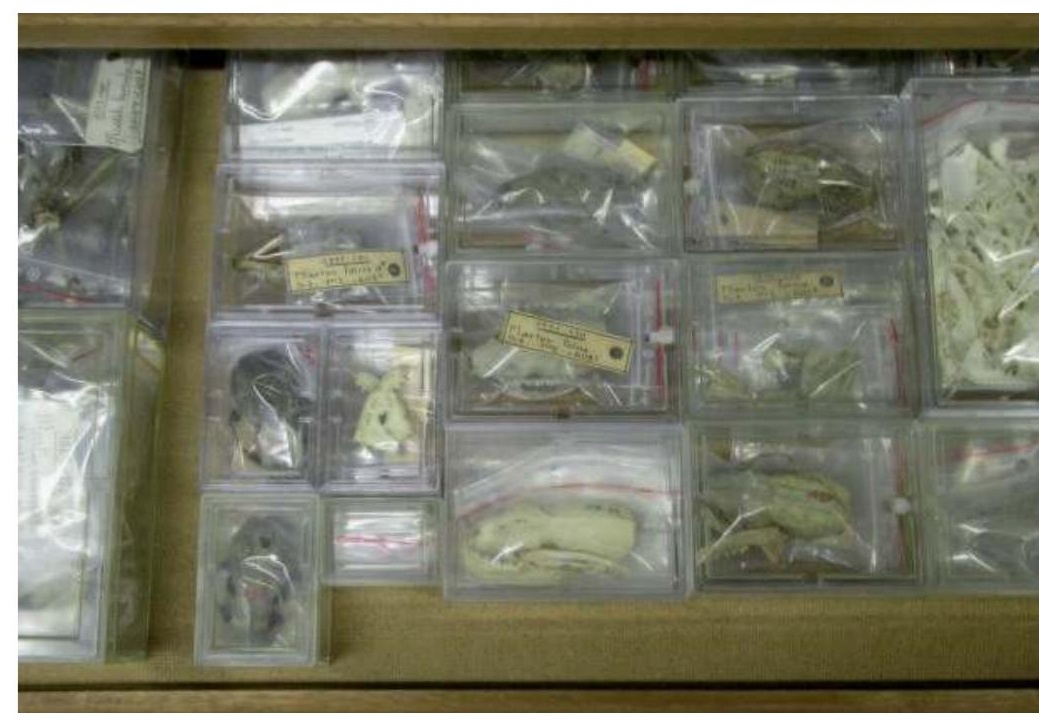

Exemple de stockage après inventaire informatique et conditionnement complet () J. Cuisin 
transparente. De manière générale, on privilégiera toujours la transparence des contenus afin de pouvoir effectuer une forme de contrôle à vue en permanence.

Lors de la consultation et de la manipulation des spécimens, les étapes de sortie et de remise du squelette dans le conditionnement sont également à considérer, pour faire face au risque de pertes par jaillissement hors du contenant. Il sera recommandé de travailler avec des plateaux à rebords, des cuvettes à fond plat, en ambiance contrastée (fond des plateaux de couleur sombre tel un gris bleu foncé permet de repérer les petits os... et les graviers !). Prévoir un temps suffisant pour la manipulation des spécimens en plus de celui dévolu à leur seul examen, a fortori en cas de travail sur des grandes séries, permettra de pallier à peu près complètement les risques de pertes à l'échelle de chaque séance.

\section{Conclusion}

Notre étude a permis de mettre en évidence et, pour la première fois, de mesurer l'influence de certains facteurs de conservation sur la complétude anatomique des spécimens des collections ostéologiques. Pour les conservateurs, ces résultats pourront contribuer à l'élaboration de stratégies efficaces de maximisation du degré d'intégrité des collections dont ils ont la charge. En tenant compte du volume des pièces, suivant les classes de taille présentées ici, il est par exemple possible d'envisager le travail de préparation ostéologique de manière différenciée, en l'organisant selon les risques que nous avons pu expliciter. De plus, notre travail pourrait être corroboré en reproduisant l'approche au sein d'autres collections ostéologiques, voire en appliquant la méthode à des collections d'une typologie totalement différente mais pouvant toutefois impliquer des problématiques similaires. L'analyse pourrait également être testée avec d'autres groupes taxonomiques. Enfin, l'étude de la notion de complétude pourrait être étendue à d'autres champs, comme par exemple la complétude systématique, la complétude typologique, la complétude géographique ou encore la complétude documentaire.

La question de la relation entre l'ancienneté des collections et la complétude anatomique des spécimens reste également ouverte. Notre étude n'a pas permis d’y répondre fermement faute de disposer de variables de contrôle suffisamment pertinentes. Des investigations supplémentaires pourraient être menées en ce sens, en définissant des variables de contrôle spécifiques pour l'ancienneté, pour le nombre cumulé des consultations et pour la quantité cumulée des actions de conservation successives. Une telle étude pourrait être menée sur des collections pour lesquelles ces informations auraient nécessairement fait l'objet d'une informatisation (date de préparation, informatisation des consultations et des prêts, enregistrement des opérations de conservation). Le cas échéant, elle permettrait de vérifier et de mesurer l'éventuel impact des actions intervenant après l'entrée en collection des spécimens.

Enfin, ce travail montre l'importance et l'intérêt de l'informatisation des collections en donnant un exemple d'une des nombreuses applications possibles, audelà de la simple consultation ponctuelle des données d'inventaires. L'informatisation détaillée des constats d'état, de même que l'effort d'informatisation des données liées à la gestion des collections, peuvent permettre de produire des corpus de données métriques analysables, autorisant alors des analyses puissantes pour répondre à des questions très concrètes qui se posent aux conservateurs.

Les auteurs tiennent à remercier Christine Lefèvre et Éric Pellé, respectivement en charge de la collection d'ostéologie et de la préparation des spécimens lors de la réalistion de ce travail.

\section{Bibliographie}

Bello, S., Thomann, A., Rabino Massa, E. et Dutour, O. Quantification de l'état de conservation des collections ostéoarchéologiques et ses champs d'application en anthropologie, Antropo, n ${ }^{\circ}$, 2003, pp. 21-37.

Cuisin, J., Bens, A., Martin, A. et Pellé, E. Organiser le stockage de grandes séries : l'exemple des artiodactyles au Muséum national d'Histoire naturelle, Conservation Restauration des Biens Culturels, n²4, 2006, pp. 21-58.

Development Core Team. A language and environment for statistical computing. Foundation for Statistical Computing, 2008, Vienna, Austria (www.R-project.org).

Gosset, W. The Probable Error of a Mean, Biometrika, vol. 6, issue 1, 1908, pp. 1-25.

Martin, A. La gestion informatisée des collections anatomiques au Muséum National d'Histoire Naturelle, La Lettre de l'OCIM, n98, 2005, pp. 21-29.

Spearman, C. Correlation calculated from faulty data, British Journal of Psychology, n³, 1910, pp. 271-295. 